\title{
LARGE GAMES WITH ONLY SMALL PLAYERS AND FINITE STRATEGY SETS
}

Abstract. Large games of kind considered in the present paper (LSFgames) directly generalize the usual concept of $n$-matrix games; the notion is related to games with a continuum of players and anonymous games with finitely many types of players, finitely many available actions and distribution dependent payoffs; however, there is no need to introduce a distribution on the set of types. Relevant features of equilibrium distributions are studied by means of fixed point, nonlinear complementarity and constrained optimization procedures in Euclidean spaces. The games are shown to fit well the voting procedures and evolutionary processes. As an example of application, we present a model of production and consumption by infinitely many households; a competitive equilibrium is obtained via a reduction to an LSF-game; the equilibrating market mechanism is modelled by actions of infinitely many small corrective powers.

1. The basic concepts. The object of our study, large games with only small players and finite strategy sets (if necessary, we refer to them as LSF-games; L for large, S for small and F for finite), directly generalize the usual concept of $n$-matrix games to nonlinear payoffs. The major equilibrium concept is found to be a fixed point of a multivalued map, a solution of a (nonlinear) complementarity problem or a solution to a (constrained) minimization problem, in all cases in a Euclidean space. An LSF-game formally describes the situation of a large number of players classified into finitely

2000 Mathematics Subject Classification: 91A06, 91A13, 91B24, 91B50, 90C33, 54H25.

Journal of Economic Literature Classification: C72, C62, E20.

Key words and phrases: large game, small players, $n$-matrix game, noncooperative game, anonymous game, measure space of players, continuum of players, Kakutani Theorem, complementarity, (Cournot-)Nash equilibrium, voting game, evolutionary models, production-consumption models, demand function, competitive equilibrium, market mechanism, invisible hand.

Research partially supported by KBN grant \# 5 H02B 00820. 
many types, with finitely many available actions and the payoffs depending only on one's own action and the distribution of the other players' actions (no large players, acting like atoms, are involved). Not surprisingly, LSF-games can be found as a mathematical skeleton hidden in the framework of some games with a continuum of players as well as in some anonymous games. This can be seen in particular when comparing the present framework with those in the papers dealing with relatively simple payoff structures, such as Schmeidler [1973], Khan and Sun [1991] or Rath [1992].

LSF-games can be applied to study evolutionary processes, also those involving many types of species, since an LSF-game depicts the behavior of a large, not necessarily homogeneous, population of anonymous individuals.

The concept of LSF-games is intended to be intermediate between structurally more complex games with a continuum (or rather with a measure space) of players and their "anonymous" rearrangements and, on the other hand, the usual $n$-matrix games, well explored from the computational point of view and popular in applications. However, an increasing number of economic models with a large number of agents and relatively simple decision structure calls for a more systematic study of their theoretical background.

We do not intend to formalize the general concept of a "large" game in the present paper, usually meaning that an infinite number of players are involved. The word "anonymous", even though appropriate, might lead to confusion with what is usually understood as an anonymous game (a distribution on the set of characteristics or types is useless in our setup).

Sections 1 and 2 deal with the basic concepts while in Section 3 we discuss some computational questions. In Sections $4-6$ we discuss the relations of LSF-games to $n$-matrix games and to games with a measure space of players, and finally, in Section 7, we show an application of LSF-games to modeling economic situations involving a large number of agents and economic powers of decentralized character.

An LSF-game is determined by a specification of positive integers $n, k^{1}, \ldots, k^{n}$ and real functions $\Phi^{1}, \ldots, \Phi^{n}$ such that $\Phi^{i}$ is defined on $V^{i} \times$ $\Delta_{k^{1}} \times \ldots \times \Delta_{k^{n}}$ (here $V^{i}$ denotes the set $\left\{1, \ldots, k^{i}\right\}$, and $\Delta_{k}$ will always denote the standard simplex with $k$ vertices $\mathbf{e}^{1(k)}=(1,0, \ldots, 0), \ldots, \mathbf{e}^{k(k)}=$ $(0,0, \ldots, 1)$, i.e. one of dimension $k-1$ in the $k$-dimensional Euclidean space). So an LSF-game can be identified with a system of integers and functions

$$
\gamma=\left(n ; k^{1}, \ldots, k^{n} ; \Phi^{1}, \ldots, \Phi^{n}\right) .
$$

The numbers $1, \ldots, n$ are types of players; for each type $i$, the numbers $1, \ldots, k^{n}$ are actions available to players of type $i$. The elements of $\Delta_{k_{1}}$ are interpreted as distributions of actions of the players of type $i$. The players themselves are not directly represented in the definition of the game. The functions $\Phi^{i}$ are the payoff functions of players of respective types. 
The intended interpretation of how an LSF-game is played is as follows. Each of infinitely many players of type 1 chooses (independently of the others) an action, which is one of the numbers $1, \ldots, k^{1}$; so the players of type 1 jointly generate a distribution of their actions which is an element of the simplex $\Delta_{k^{1}}$; similarly, the players of any other type $i$ choose among the numbers $1, \ldots, k^{i}$ and jointly generate a distribution $\mathbf{p}^{i}$ of their actions which is an element of $\Delta_{k^{i}}$. If a specific player of type $i$ has chosen an individual action $j \in\left\{1, \ldots, k^{i}\right\}$, then his payoff is $\Phi^{i}\left(j ; \mathbf{p}^{1}, \ldots, \mathbf{p}^{n}\right)$; we understand that an individual player's action does not affect the distribution of the actions of other players of his type (nor of other types).

We say that an LSF-game is continuous whenever all functions $\Phi^{i}(j ; \cdot)$, $i=1, \ldots, n, j \in V^{i}$, are continuous. It is affine whenever all these functions are affine (more precisely: have affine extensions to the whole Euclidean space $\left.\mathbb{R}^{k^{1}+\ldots+k^{n}}\right)$.

2. Equilibria. The basic equilibrium concept for LSF-games that will be studied throughout the paper reflects the case where no substantial fraction of the players can increase their individual payoffs.

A distribution of actions $\mathbf{p}=\left(\mathbf{p}^{1}, \ldots, \mathbf{p}^{n}\right)$ in an LSF-game is said to be an equilibrium (or to be an equilibrated distribution) whenever there exist real numbers $C^{1}, \ldots, C^{n}$ such that

$$
\begin{array}{ll}
\Phi^{i}\left(j ; \mathbf{p}^{1}, \ldots, \mathbf{p}^{n}\right) \leq C^{i} & \text { for } i=1, \ldots, n \text { and } j \in V^{i}, \\
\Phi^{i}\left(j ; \mathbf{p}^{1}, \ldots, \mathbf{p}^{n}\right)=C^{i} & \text { for } i=1, \ldots, n \text { and } j \in \operatorname{supp} \mathbf{p}^{i} .
\end{array}
$$

For an equilibrium $\mathbf{p}$, the corresponding numbers $C^{i}$ (which are unique) are called payoff levels (of respective types $i$ at $\mathbf{p}$ ).

So a distribution $\mathbf{p}$ forms an equilibrium whenever (almost) all players who are of the same type $i$ get the same payoff $C^{i}$ at $\mathbf{p}$; actions that are (essentially) not used ( $j$ such that $\left.p_{j}^{i}=0\right)$ cannot yield higher payoffs, since this would tempt the individuals to switch their actions to such a $j$, contradicting the common understanding of equilibrium.

The problem of computing all equilibria for an LSF-game is clearly equivalent to solving the following problem ("E" for equalizing):

(E) Find $\mathbf{p}=\left(\mathbf{p}^{1}, \ldots, \mathbf{p}^{n}\right) \in \Delta_{k^{1}} \times \ldots \times \Delta_{k^{n}}$ and real numbers $C^{1}, \ldots, C^{n}$ satisfying (1).

2.1. Theorem. If all functions $\Phi^{i}(j ; \cdot), i=1, \ldots, n, j \in V^{i}$, are continuous then the problem (E) has a solution, hence every continuous LSF-game has an equilibrium.

Proof. Define a correspondence $H$ from $D=\Delta_{k_{1}} \times \ldots \times \Delta_{k_{k}}$ to subsets of $D$ by letting 


$$
\begin{aligned}
H^{i}(\mathbf{p}) & :=\operatorname{co}\left\{\mathbf{e}^{t\left(k^{i}\right)} \mid t \in \operatorname{Argmax}_{j \in V^{i}} \Phi^{i}(j ; \mathbf{p})\right\} \quad \text { for } i=1, \ldots, n, \\
H(\mathbf{p}) & :=H^{1}(\mathbf{p}) \times \ldots \times H^{n}(\mathbf{p}) .
\end{aligned}
$$

Since $D$ is nonempty convex and compact, and $H$ is upper semicontinuous (standard verification) and its values are simplices, it follows from the Kakutani Theorem that $H$ has a fixed point $\mathbf{p}$. This $\mathbf{p}$, together with immediately found constants $C^{i}$, gives a solution to $(\mathbf{E})$.

We shall now introduce some numerical characteristics of distributions for a given LSF-game, which are defined for a type $i$ of players and for a pair of distributions: a distribution $\mathbf{p}^{i}$ of actions of the players of type $i$ and a "total" distribution of actions $\mathbf{q}=\left(\mathbf{q}^{1}, \ldots, \mathbf{q}^{n}\right)$ of all types. The symbols "ess inf ", "aver" and "ess sup" stand for the essential infimum, average and essential supremum of the payoffs of the players of type $i$ at $\mathbf{q}$, but taken with respect to a distribution $\mathbf{p}^{i}$. Some notation, which may now appear excessive, will turn out to be useful in Section 5.

Formally, for any LSF-game

$$
\gamma=\left(n ; k^{1}, \ldots, k^{n} ; \Phi^{1}, \ldots, \Phi^{n}\right)
$$

and any distribution $\mathbf{q}=\left(\mathbf{q}^{1}, \ldots, \mathbf{q}^{n}\right)$ we define, for $i=1, \ldots, n$ and $\mathrm{a}$ distribution $\mathbf{p}^{i}=\left(p_{1}^{i}, \ldots, p_{k^{i}}^{i}\right)$, the numbers ("supp" stands for support):

$$
\begin{aligned}
\operatorname{essinf}_{\mathbf{p}^{i}} \Phi^{i}[\mathbf{q}] & :=\min \left\{\Phi^{i}(j, \mathbf{q}) \mid j \in \operatorname{supp} \mathbf{p}^{i}\right\} \\
\operatorname{aver}_{\mathbf{p}^{i}} \Phi^{i}[\mathbf{q}] & :=\left\langle\Phi^{i}[\mathbf{q}] ; \mathbf{p}^{i}\right\rangle=\sum_{j \in V^{i}} \Phi^{i}(j, \mathbf{q}) \cdot p_{j}^{i} \\
\operatorname{ess} \sup _{\mathbf{p}^{i}} \Phi^{i}[\mathbf{q}] & :=\max \left\{\Phi^{i}(j, \mathbf{q}) \mid j \in \operatorname{supp} \mathbf{p}^{i}\right\} \\
\max \Phi^{i}[\mathbf{q}] & :=\max \left\{\Phi^{i}(j, \mathbf{q}) \mid j \in V^{i}\right\}
\end{aligned}
$$

We obviously always have

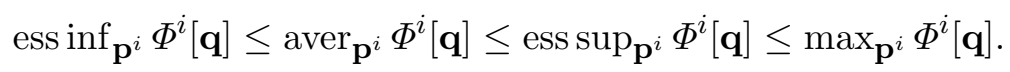

2.2. Proposition. For any LSF-game $\gamma$ and any distribution $\mathbf{p}$ the following conditions are equivalent:

(i) $\mathbf{p}$ is an equilibrium;

(ii) for every type $i, \operatorname{essinf}_{\mathbf{p}^{i}} \Phi^{i}[\mathbf{p}]=\operatorname{aver}_{\mathbf{p}^{i}} \Phi^{i}[\mathbf{p}]$ while $\operatorname{ess~sup}_{\mathbf{p}^{i}} \Phi^{i}[\mathbf{p}]=$ $\max _{\mathbf{p}^{i}} \Phi^{i}[\mathbf{p}]$

(iii) for every type $i$, $\operatorname{aver}_{\mathbf{p}^{i}} \Phi^{i}[\mathbf{p}]=\max _{\mathbf{p}^{i}} \Phi^{i}[\mathbf{p}]$;

(iv) for every type $i$, ess inf $\mathbf{p}^{i} \Phi^{i}[\mathbf{p}]=\max _{\mathbf{p}^{i}} \Phi^{i}[\mathbf{p}]$.

Proof. This follows easily from the definitions.

3. Computation of equilibria. In this section we show how to reduce the problem of computing all equilibria of an LSF-game to a standard 
(nonlinear) complementarity problem (see e.g. Isac [1992] for a survey) or to a constrained minimization problem. Both procedures also work well in the case of affine LSF-games where they roughly reduce to procedures well known for $n$-matrix games, originated by the papers of Lemke and Howson [1964] and Nikaido and Isoda [1955].

In this section we also study the simplest and very intuitive one type-two actions case and give an example interpreted in terms of a voting scheme.

The problem $(\mathbf{E})$ is obviously equivalent to the following (generally nonlinear) complementarity problem:

(CP) Given natural numbers $n, k^{1}, \ldots, k^{n}$ and real-valued functions $\Phi^{i}$ : $V^{i} \times \Delta_{k_{1}} \times \ldots \times \Delta_{k_{n}} \rightarrow \mathbb{R}, i=1, \ldots, n$, find $\left(\mathbf{p}^{1}, \ldots, \mathbf{p}^{n}\right) \in \Delta_{k_{1}} \times$ $\ldots \times \Delta_{k_{n}}$ and real constants $C^{1}, \ldots, C^{n}$ such that

$$
\Phi^{i}\left(j ; \mathbf{p}^{1}, \ldots, \mathbf{p}^{n}\right)-C^{i} \leq 0 \quad \text { for all } i=1, \ldots, n \text { and } j \in V^{i}
$$

and

$$
\sum_{i=1, \ldots, n, j \in V^{i}}\left(\Phi^{i}\left(j ; \mathbf{p}^{1}, \ldots, \mathbf{p}^{n}\right)-C^{i}\right) \cdot p_{j}^{i}=0
$$

or, in the more familiar shorthand form,

$\left(\mathbf{C P}^{\prime}\right) \quad$ Find $(\mathbf{p}, \mathbf{C}) \in \Delta_{k^{1}} \times \ldots \times \Delta_{k^{n}} \times \mathbb{R}^{n}$ such that

$$
F(\mathbf{p}, \mathbf{C}) \leq \mathbf{0} \quad \text { and } \quad\langle F(\mathbf{p}, \mathbf{C}) ; \mathbf{p}\rangle=0
$$

where $\langle\cdot ; \cdot\rangle$ denotes the inner product and the function $F$ is defined by $F(\mathbf{p}, \mathbf{C}):=$

$$
\left(\Phi^{1}(1 ; \mathbf{p})-C^{1}, \ldots, \Phi^{1}\left(k^{n} ; \mathbf{p}\right)-C, \ldots, \Phi^{n}(1 ; \mathbf{p})-C^{n}, \ldots, \Phi^{n}\left(k^{n} ; \mathbf{p}\right)-C^{n}\right) .
$$

So we have:

3.1. Proposition. A vector $\left(\mathbf{p}^{1}, \ldots, \mathbf{p}^{n}\right)$ is an equilibrium for an LSFgame $\gamma$, with the corresponding payoff levels $\left(C^{1}, \ldots, C^{n}\right)$, if and only if the system $\left(\mathbf{p}^{1}, \ldots, \mathbf{p}^{n} ; C^{1}, \ldots, C^{n}\right)$ solves the problem $(\mathbf{C P})$.

Notice that whenever the functions $\Phi^{i}$ are uniformly bounded, by some $m$ from below and $M$ from above, then the range of the variables $C^{i}$ can be restricted, if necessary for any reasons, to the interval $[m, M]$.

If an equilibrium with some particular properties is to be found, e.g. one maximizing or minimizing the value of a function $G(\mathbf{p}, C)$, like $G(\mathbf{p}, C)=$ $\sum_{i} C^{i}$, the problem would be equivalent to solving the problem of maximizing or minimizing $G(\mathbf{p}, C)$ subject to the constraints (2).

An alternative approach to computing equilibria is to investigate a single real function, defined for all distributions, whose values happen to be zero just when the distribution is equilibrated. For an LSF-game

$$
\gamma=\left(n ; k^{1}, \ldots, k^{n} ; \Phi^{1}, \ldots, \Phi^{n}\right)
$$


we define the measure of disequilibration as a function $\Theta_{\gamma}: \Delta_{k^{1}} \times \ldots \times \Delta_{k^{n}}$ $\rightarrow \mathbb{R}$ such that for all $\left(\mathbf{p}^{1}, \ldots, \mathbf{p}^{n}\right) \in \Delta_{k^{1}} \times \ldots \times \Delta_{k^{n}}$,

$$
\Theta_{\gamma}(\mathbf{p}):=\sum_{i=1}^{n}\left(\max \Phi^{i}[\mathbf{p}]-\underset{\mathbf{p}^{i}}{\operatorname{aver}} \Phi^{i}[\mathbf{p}]\right) .
$$

3.2. TheOrEm. The measure of disequilibration is nonnegative. For any distribution $\mathbf{p}, \Theta_{\gamma}(p)=0$ if and only if $\mathbf{p}$ is an equilibrium. If $\gamma$ is continuous then so is $\Theta_{\gamma}$.

Proof. This follows from (2) and Proposition 2.2.

So the problem of finding all equilibria for an LSF-game $\gamma$ reduces to that of finding all zeros (equivalently: all global minima) of the function $\Theta_{\gamma}$.

There is no need to go any further into details of how to practically solve a problem of this kind or how to solve a complementarity problem $(\mathbf{C P})$ or $\left(\mathbf{C P}^{\prime}\right)$ as the existing literature of the subject is large and exhaustive while the choice of a specific method would heavily depend on the kind of the functions $\Phi^{i}$ involved. Methods allowing one to attack directly the problems formulated in this section (optimization problems on a simplotope, a product of simplices) were elaborated in the eighties by Doup, Van der Elzen, Van der Heyden, Van der Laan, Talman and others (Doup et al. [1983], [1987] (three items) and Van der Laan et al. [1982], [1987]).

The search for equilibrated distributions is specially instructive in the simplest nontrivial special case, where there is just one type of players endowed with two actions; hence the game is entirely determined by two real functions $\varphi_{1}, \varphi_{2}$ defined on the unit interval $[0,1]\left(\varphi_{j}\right.$ stands for $\Phi^{1}(j ; \cdot)$ in our previous notation). Then, by definition, $(p, 1-p)$ is an equilibrium in just three cases:

- when $(p, 1-p)=(1,0)$ while $\varphi_{1}(0) \geq \varphi_{2}(0)$;

- when $(p, 1-p)=(0,1)$ while $\varphi_{2}(1) \geq \varphi_{1}(1)$;

- when $\varphi_{1}(p)=\varphi_{2}(p)$.

Hence, to find all equilibria in this case, it is sufficient to look at the graphs of $\varphi_{1}$ and $\varphi_{2}$, verify their behavior at the endpoints of the interval $[0,1]$ and find all intersection points of the two graphs.

This very same reasoning also illustrates a straightforward and natural proof of Theorem 2.1 in this case: if $\varphi_{2}(0)>\varphi_{1}(0)$ and $\varphi_{1}(1)>\varphi_{2}(1)$, the (graphs of the) continuous functions $\varphi_{1}$ and $\varphi_{2}$ must, by the Mean Value Theorem, intersect at some $p^{*}$ between 0 and 1 , which yields an equilibrium.

Some voting procedures can be represented as LSF-games in the manner depending on the specific election rules:

3.4. ExAmple. Suppose that the electorate has been classified into just one type, and one option among $k$ should be chosen. Let the payoffs be defined by $\Phi(j ; \mathbf{p})=p_{j}$ for $j=1, \ldots, k$, i.e. if I vote for party number $j$ 
then my payoff is the fraction of votes collected by this party (the rule is cynical or indifferent: I do not care which particular party to support but I do care to be with one gaining the largest fraction of votes). It is easy to check (e.g. by computing the zeros of the measure of disequilibration $\left.\Theta(\mathbf{p}):=\max _{i} p_{i}-\left(p_{1}^{2}+p_{2}^{2}+\ldots+p_{k}^{2}\right)\right)$ that a distribution $\mathbf{p}$ is equilibrated if and only if there is an integer $1 \leq k_{0} \leq k$ such that $p_{j}=k_{0}^{-1}$ for exactly $k_{0}$ indices $j$ and otherwise $p_{j}=0$.

4. Relations to $n$-matrix games. The idea of interpreting the payoffs associated with playing a mixed strategy in an $n$-matrix game in terms of an average payoff while playing the game many times and using the pure strategies with frequencies close to probabilities assigned by this mixture, is nearly as old as the concept of mixed strategy itself. In many models, especially those which concern the adaptation behavior or learning process (see e.g. Malawski [1989]), there even appears a large population of players playing the same game and hence provoking the other players' reaction. This notion of "large" has been formalized in this context in various ways, often also involving the presence of a continuum of players.

However, in many contexts (cf. Example 3.4 or the model in Section 7) there is no necessity to assume the affine dependence of the payoffs on frequencies of the use of pure strategies by other players, as is usually done in the case of $n$-matrix games.

In the present section we shall formally represent an $n$-matrix game as an LSF-game and show that the Nash equilibria of the former exactly correspond to the equilibrated distributions of the latter.

The usual $n$-matrix game $A$ is specified by a system of $n$ positive integers $k^{1}, \ldots, k^{n}, k^{i}$ being the number of the $i$ th player's pure strategies and by a system of payoff functions $A^{i}: V^{1} \times \ldots \times V^{n} \rightarrow \mathbb{R}$, where $V^{i}$ denotes the set $\left\{1, \ldots, k^{i}\right\}$. These functions are usually identified with $n$-dimensional $k^{1} \times \ldots \times k^{n}$-matrices.

The payoff of player $i$ while players $1, \ldots, n$ (including himself) use their respective mixed strategies $\mathbf{p}^{1}, \ldots, \mathbf{p}^{n}$ is then, as usual, the number

$$
\widetilde{A}^{i}\left(\mathbf{p}^{1}, \ldots, \mathbf{p}^{n}\right):=\sum_{j^{1} \in V^{1}, \ldots, j^{n} \in V^{n}} p_{j^{1}}^{1} \ldots p_{j^{n}}^{n} A^{i}\left(j^{1}, \ldots, j^{n}\right) .
$$

The corresponding LSF-game $\gamma(\mathbf{A})$, which may be called a standard extension of $\mathbf{A}$, is determined by the system of integers $n, k^{1}, \ldots, k^{n}$ and the functions

$$
\Phi^{i}\left(j ; \mathbf{p}^{1}, \ldots, \mathbf{p}^{n}\right):=\widetilde{A}^{i}\left(\mathbf{p}^{1}, \ldots, \mathbf{p}^{i-1}, \mathbf{e}^{j\left(k^{i}\right)}, \mathbf{p}^{i+1}, \ldots, \mathbf{p}^{n}\right) .
$$

The LSF-game $\gamma(\mathbf{A})$ can be identified with the initial $n$-matrix game $\mathbf{A}$, since it includes complete information necessary to identify $\mathbf{A}$ itself: the 
$n$-matrix game $\mathbf{A}$ whose extension is the given LSF-game $\gamma(\mathbf{A})$ (with payoff functions $\Phi^{i}$ ) is immediately found to be

$$
A^{i}\left(j^{1}, \ldots, j^{n}\right):=\Phi^{i}\left(j^{i} ; \mathbf{e}^{j^{1}\left(k^{1}\right)}, \ldots, \mathbf{e}^{j^{i-1}\left(k^{i-1}\right)}, \mathbf{p}^{i}, \mathbf{e}^{j^{i+1}\left(k^{i+1}\right)}, \ldots, \mathbf{e}^{j^{n}\left(k^{n}\right)}\right),
$$

$j=1, \ldots, n, j^{i} \in V^{i} ; \mathbf{p}^{i}$ is arbitrary. In this sense LSF-games can be regarded as generalizing the concept of $n$-matrix games.

So our next objective is to determine which LSF-games are standard extensions of some $n$-matrix games.

4.1. TheOREM. An LSF-game $\gamma$ with $n$ types of players is a standard extension of an n-matrix game if and only if it is affine and, for $i=1, \ldots, n$ and $j=1,2, \ldots, k^{i}$, the function $\Phi^{i}\left(j ; \mathbf{p}^{1}, \ldots, \mathbf{p}^{i}, \ldots, \mathbf{p}^{n}\right)$ is actually independent of $\mathbf{p}^{i}$.

Proof. This is standard; we skip the details.

Finally, we should compare the equilibrated distributions in an LSF-game with the Nash equilibria in the corresponding $n$-matrix game.

As usual, a system $\left(\mathbf{p}^{1}, \ldots, \mathbf{p}^{n}\right)$ of strategies is called a Nash equilibrium for an $n$-matrix game $\mathbf{A}$ whenever no player $i$ has a strategy $\widetilde{\mathbf{p}}^{i}$ such that

$$
A^{i}\left(\mathbf{p}^{1}, \ldots, \mathbf{p}^{i-1}, \widetilde{\mathbf{p}}^{i}, \mathbf{p}^{i+1}, \ldots, \mathbf{p}^{n}\right)>A^{i}\left(\mathbf{p}^{1}, \ldots, \mathbf{p}^{n}\right) .
$$

4.2. TheOREM. A vector $\mathbf{p}$ is an equilibrium for the standard extension $\gamma(\mathbf{A})$ of an n-matrix game $\mathbf{A}$ if and only if it is a Nash equilibrium for $\mathbf{A}$.

Proof. This follows easily from Lemma 4.3 below, which is a sort of "common knowledge", but it is also easily verifiable.

4.3. Lemma. A vector $\mathbf{p}=\left(\mathbf{p}^{1}, \ldots, \mathbf{p}^{n}\right)$ is a Nash equilibrium for an $n$-matrix game $A$ if and only if for each player $i$ and each of his pure strategies $j$,

$$
\widetilde{A}^{i}\left(\mathbf{p}^{1}, \ldots, \mathbf{p}^{i-1}, \mathbf{e}^{j\left(k^{i}\right)}, \mathbf{p}^{i+1}, \ldots, \mathbf{p}^{n}\right) \leq \widetilde{A}^{i}\left(\mathbf{p}^{1}, \ldots, \mathbf{p}^{n}\right)
$$

and

$$
\widetilde{A}^{i}\left(\mathbf{p}^{1}, \ldots, \mathbf{p}^{i-1}, \mathbf{e}^{j\left(k^{i}\right)}, \mathbf{p}^{i+1}, \ldots, \mathbf{p}^{n}\right)=\widetilde{A}^{i}\left(\mathbf{p}^{1}, \ldots, \mathbf{p}^{n}\right) \text { whenever } p_{j}^{i} \neq 0 .
$$

We should warn of possible misunderstandings: the ordinary definition of Nash equilibria for an $n$-matrix game might suggest its extension to LSF-games in the following manner: a distribution $\mathbf{p}=\left(\mathbf{p}^{1}, \ldots, \mathbf{p}^{n}\right)$ is in some sense equilibrated (e.g. stable against reaction of players of a single type) whenever there is no type $i$ and no distribution $\widetilde{\mathbf{p}}^{i}$ such that $\operatorname{aver}_{\widetilde{\mathbf{p}}^{i}} \Phi\left[\mathbf{p}^{1}, \ldots, \mathbf{p}^{i-1}, \widetilde{\mathbf{p}}^{i}, \mathbf{p}^{i+1}, \ldots, \mathbf{p}^{n}\right]>\operatorname{aver}_{\mathbf{p}^{i}} \Phi[\mathbf{p}]$. This definition yields an equilibrium concept entirely different from that of the present paper, 
although the two coincide for LSF-games which are standard extensions of $n$-matrix games (for continuous LSF-games every distribution with the above property is an equilibrium).

5. Relations to evolutionary game-theoretic models. Situations involving a large number of identically characterized players are often modeled, in the evolutionary context, after Maynard Smith [1982], as two-person symmetric games; models involving two types of individuals were first considered by Taylor [1979], Schuster et al. [1983] and systematically studied by Cressman [1995]; cf. also Ritzberger and Weibull [1995] for the case of $n$ types.

An alternative approach can be offered in the scope of LSF-games, allowing for nonlinear payoffs but also for a uniform treatment of the behavior of single- and multi-population $(n)$ cases (the latter is often modelled as an $n$-person, not $(n+1)$-person or $2 n$-person game, which is an unnecessary conceptual differentiation, see e.g. Weibull [1995], Chapters 2-5). We give below a definition of evolutionary stable distributions for an LSF-game and we compare it with the standard one used for symmetric bimatrix games. We define the concept in the general case of $n$ types of players, but we compare it with the standard one only in the case of one type (although it also extends an analogous definition for a four-matrix model of two populations as analysed by Cressman [1995]).

This section is addressed to the readers who may expect some relations between LSF-games and familiar concepts in evolutionary game theory; we answer only the most basic question concerning evolutionary stable strategies (ESS) but we postpone a more detailed discussion.

A bimatrix game $\mathbf{A}=\left(A^{1}, A^{2}\right)$, with the same set of pure strategies $V=\{1, \ldots, k\}$ for both players, is symmetric whenever $A^{1}\left(j, j^{\prime}\right)=A^{2}\left(j^{\prime}, j\right)$ for any $j, j^{\prime}$ in $V$ (so $A^{2}$ is actually redundant in notation for symmetric games). A vector $\mathbf{p} \in \Delta_{k}$ is an evolutionary stable strategy (ESS) for $\mathbf{A}$ if it is a Nash equilibrium for $\mathbf{A}$ and for every $\mathbf{q} \in \Delta_{k}$ different from $\mathbf{p}$, $\widetilde{A}^{1}(\mathbf{q}, \mathbf{p})=\widetilde{A}^{1}(\mathbf{p}, \mathbf{p})$ implies $\widetilde{A}^{1}(\mathbf{q}, \mathbf{q})<\widetilde{A}^{1}(\mathbf{p}, \mathbf{q})$ (we use the notation of the previous section).

An LSF-game $\gamma^{*}(\mathbf{A})$ associated with $\mathbf{A}$ (not to be confused with the standard extension $\gamma(\mathbf{A})$ ) as defined in Section 4!) is determined by the integer $k$ and a function $\Psi$ (the superscript ${ }^{1}$ indicating the type is skipped) defined by

$$
\Psi(j ; \mathbf{p}):=\widetilde{A}^{1}\left(\mathbf{e}^{j(k)}, \mathbf{p}\right) .
$$

Clearly, $\gamma^{*}(\mathbf{A})$ is affine.

An equilibrium $\mathbf{p}=\left(\mathbf{p}^{1}, \ldots, \mathbf{p}^{n}\right)$ for an LSF-game $\gamma$ will be called evolutionary stable whenever, for every distribution $\mathbf{q}=\left(\mathbf{q}^{1}, \ldots, \mathbf{q}^{n}\right)$ such that 
$\operatorname{supp}\left(\mathbf{q}^{i}\right) \subseteq \operatorname{supp}\left(\mathbf{p}^{i}\right)$ for all $i=1, \ldots, n$, we have $\operatorname{aver}_{\mathbf{q}^{i}} \Psi^{i}[\mathbf{q}]<\operatorname{aver}_{\mathbf{p}^{i}} \Psi^{i}[\mathbf{q}]$ for some $i$ (see Section 2 for notation).

The definition above may be meaningfully modified in various ways by replacing some "aver" symbols by "ess sup" or "ess inf" and differentiating the quantifiers involving $i$.

5.1. TheOREM. Let $\gamma^{*}(\mathbf{A})$ be the LSF-game associated with a bimatrix game $\mathbf{A}=\left(A^{1}, A^{2}\right)$ with $k$ pure strategies of each player and let $\mathbf{p}$ be a distribution for $\gamma^{*}(\mathbf{A})$. Then $\mathbf{p}$ is an ESS for $\mathbf{A}$ if and only if it is an evolutionary stable distribution for $\gamma^{*}(\mathbf{A})$.

Proof. Routine.

6. Relations to games with a continuum of players. We have already noted the interpretation of LSF-games as involving an infinite population of players. This interpretation can be more formally justified by relating LSF-games to some games with a continuum of players involving finitely many types and having a finite number of available strategies, namely those in which every player's payoff depends only on his own strategy and a distribution of the other players' choice of strategies. A comparison of this kind is offered in this section.

A rigorous formal definition of noncooperative games with a continuum of players, also often referred to as games with a measure space of players, was given by Schmeidler [1973]. This concept was then developed by Khan [1985, 1989], Balder [1990, 1995], Rath [1992], Flåm and Wieczorek [1996], Khan and Rustichini [1991, 1993] and others. The anonymous situations have been considered by Mas-Colell [1984], Rath [1992], Balder [1990] and others, the idea going back to Hart, Hildenbrand and Kohlberg [1974]. An in-depth study of relations of the general case to the anonymous case was given by Rath [1995]. We stress that the essential difference between LSFgames and similar anonymous games is that the former do not involve any a priori distribution of the players of various types.

Theorem 2.1 in this paper can also be derived, with some auxiliary constructions, from quite general results of Schmeidler [1973] and Rath [1992] or even Mas-Colell [1984]; however, the proof given in Section 2 does not involve any elements unnecessary in the present context and it also directly suggests computational procedures to get an equilibrated distribution.

A game with a continuum of players or, more properly, a game with a measure space of players, is given by a specification of the players, usually identified with elements of a normed measure space $(T, \mathcal{T}, \mu)$, the players' nonempty strategy sets $S^{t}, t \in T$, assumed to be all included in some set $S$ (usually for technical reasons equipped with a $\sigma$-field $\Sigma$ ) and the players' payoff functions. To define the latter, we need the notion of a strategy profile: 
it is a measurable function $s: T \rightarrow S$ such that $s(t) \in S^{t}$ for all $t \in T$. The payoff function of player $t, u^{t}\left(\sigma^{t}, s\right)$, depends on the player's own choice of strategy $\sigma^{t} \in S^{t}$ and the strategy profile $s$. We assume that $u^{t}\left(\sigma^{t}, s\right)=$ $u^{t}\left(\sigma^{t}, s^{\prime}\right)$ whenever the profiles $s$ and $s^{\prime}$ are measure equivalent. Hence, a game with a measure space of players is identified with a system

$$
\Gamma=\left((T, \mathcal{T}, \mu),\left(S^{t} \mid t \in T\right),(S, \Sigma),\left(u^{t} \mid t \in T\right)\right) .
$$

Measurable sets of players of measure zero are referred to as negligible. A strategy profile $s$ is said to form a Cournot-Nash equilibrium if the set of players $t$ who can find a strategy $\sigma^{t} \in S^{t}$ such that $u^{t}\left(\sigma^{t}, s\right)>u^{t}(s(t), s)$ is negligible.

We say that players are of the same type whenever they have the same strategy sets and payoff functions.

6.1. TheOrem (equal treatment). At a Cournot-Nash equilibrium the payoff of almost all players of the same type is equal (even though they may use different strategies).

Proof. To the contrary, suppose that, for a Cournot-Nash equilibrium $s$ and a constant $C, u^{t}(s(t), s)>C$ for all players $t$ in a nonempty set $B$ of players while $u^{t}(s(t), s) \leq C$ for all players $t$ in a nonnegligible set $B^{\prime}$. Then the members of $B^{\prime}$ could improve their payoffs just by switching their strategies to $s\left(t_{0}\right)$, where $t_{0}$ is any element of $B$.

Suppose now that in a game $\Gamma$ there are, possibly except for a negligible set of players, only finitely many types of players endowed with finite strategy sets.

For such a game $\Gamma$, we label the types of players by $1, \ldots, n$ and denote by $T^{i}$ the set of all players of type $i$ (we assume that these sets are measurable and $\left.\mu\left(T^{i}\right)>0\right)$. Let $1, \ldots, k^{i}$ label elements in the strategy set $S^{i}$ of players of type $i$. Neglecting the players in a negligible set we may then assume that $S$ is finite (with $\Sigma$ being the field of all subsets). For any strategy profile $s$, any type $i$ and any $j \in S^{i}$, we denote by

$$
\kappa_{s}(i, j)=\mu\left(\left\{t \in T^{i} \mid s(t)=j\right\}\right) / \mu\left(T^{i}\right)
$$

the frequency of players of type $i$ who use strategy $j$ at profile $s$. The function $\kappa_{s}$ will also be referred to as the distribution of $s$. We also set

$$
K_{s}(i):=\left(\kappa_{s}(i, 1), \ldots, \kappa_{s}\left(i, k^{i}\right)\right) .
$$

We shall say that a game with a measure space of players is of finite type whenever there are finitely many types of players, endowed with finite strategy sets and, for every player $t$ of type $i$, his payoff function has the form

$$
u^{t}\left(\sigma^{t}, s\right):=\Phi^{i}\left(\sigma^{t} ; K_{s}(1), \ldots, K_{s}(n)\right),
$$


i.e. every player's payoff only depends on his own choice of action and the distribution of the other players' actions.

In that case we define an LSF-game $\gamma$ corresponding to $\Gamma$ as

$$
\gamma:=\left(n ; k^{1}, \ldots, k^{n} ; \Phi^{1}, \ldots, \Phi^{n}\right),
$$

where all numbers and functions above are those already considered for $\Gamma$.

6.2. TheOREM. Let an LSF-game $\gamma$ correspond to a game $\Gamma$ with a measure space of players, of finite type, and let $s$ be any strategy profile for $\Gamma$. Then $s$ is a Cournot-Nash equilibrium for $\Gamma$ if and only if $\mathbf{K}_{s}:=\left(K_{s}(1), \ldots, K_{s}(n)\right)$ is an equilibrium distribution for $\gamma$.

Proof. In view of Theorem 6.1, this a standard verification.

Conversely, for every LSF-game $\gamma$, one can easily find a game $\Gamma$ with a measure space of players, of finite type, to which $\gamma$ corresponds. Suppose that $\gamma=\left(n ; k^{1}, \ldots, k^{n} ; \Phi^{1}, \ldots, \Phi^{n}\right)$. Then one of the simplest instances of such a game $\Gamma=\left((T, \mathcal{T}, \mu),\left(S^{t} \mid t \in T\right),(S, \Sigma),\left(u^{t} \mid t \in T\right)\right)$ is constructed as follows: we let $(T, \mathcal{T}, \mu)$ be the interval $[0,1)$ with Lebesgue measurable subsets and Lebesgue measure; $S^{t}=\left\{1, \ldots, k^{i}\right\}$ whenever $t \in[(i-1) / n, i / n)$; $S=\left\{1, \ldots, \max _{i} k^{i}\right\}$ and $\Sigma$ consists of all subsets of $S$; finally, we define $u^{t}$ for each player $t$ in $[(i-1) / n, i / n)$ by letting

$$
u^{t}\left(\sigma^{t}, s\right):=\Phi^{i}\left(\sigma^{t} ; K_{s}(1), \ldots, K_{s}(n)\right) .
$$

\section{An application: a household economy with many agents and} decentralized market mechanism. As an example of application, we shall consider an economy in which there are infinitely many agents, classified into $n$ types. For each type $i$, a positive number $q^{i}$ evaluates the volume or measure of the set of all agents of type $i$ (for convenience it may be assumed that $\sum_{i=1}^{n} q^{i}=1$ ). Each agent, of any type, has a choice between $k$ kinds of activity. Undertaking the $j$ th activity by a type $i$ agent results in producing $r_{j}^{i}$ units of the $j$ th commodity, and gives the agent an income of $I=r_{j}^{i} \pi_{j}$, where $\pi_{j}$ is the current price of the $j$ th commodity. The nonnegative number $r_{j}^{i}$ is a coefficient of efficiency; we also set, for each type $i$, $\bar{r}^{i}=\max _{j} r_{j}^{i}$. The consumption of an agent of type $i$ is determined by means of a demand function $d^{i}=d^{i}(I, \pi)$, where $I$ denotes the individual income while $\pi=\left(\pi_{1}, \ldots, \pi_{k}\right)$ is the system of prevailing prices. We assume that $d^{i}:\left[0, \bar{r}^{i}\right] \times \Delta_{k} \rightarrow \mathbb{R}_{+}^{k}$ and, for all values of the arguments, $\left\langle d^{i}(I, \pi) ; \pi\right\rangle \leq I$ (prices are assumed to be normalized and hence the individual income of an agent of type $i$ never exceeds $\bar{r}^{i}$ ).

Hence, the full characteristic of the type of an agent is given by a vector $\mathbf{r}^{i}=\left(r_{1}^{i}, \ldots, r_{k}^{i}\right)$ of nonnegative numbers and the demand function $d^{i}$. 
The description of an economy $\mathcal{E}$ is complete with characteristics of types and a vector $\mathbf{q}=\left(q^{1}, \ldots, q^{n}\right)$ of volumes of the respective types.

Suppose that the distribution of actions of agents of type $i$ is $\mathbf{p}^{i}=$ $\left(p_{1}^{i}, \ldots, p_{k}^{i}\right) \in \Delta_{k}$. Then the aggregated demand is given by

$$
\mathbf{d}\left(\mathbf{p}^{1}, \ldots, \mathbf{p}^{n} ; \pi\right):=\sum_{i=1}^{n} q^{i} \sum_{j=1}^{k} d^{i}\left(r_{j}^{i} \pi_{j}, \pi\right) \cdot p_{j}^{i},
$$

while the aggregated supply is

$$
\mathbf{s}\left(\mathbf{p}^{1}, \ldots, \mathbf{p}^{n}\right):=\left(\sum_{i=1}^{n} q^{i} r_{1}^{i} p_{1}^{i}, \ldots, \sum_{i=1}^{n} q^{i} r_{k}^{i} p_{k}^{i}\right) .
$$

The competitive equilibrium is defined as an $(n+1)$-tuple $\left(\mathbf{p}^{1}, \ldots, \mathbf{p}^{n} ; \pi\right)$, of distributions of actions of respective types of agents and a price system, satisfying the physical balance (coordinatewise) inequality

$$
\mathbf{d}\left(\mathbf{p}^{1}, \ldots, \mathbf{p}^{n} ; \pi\right) \leq \mathbf{s}\left(\mathbf{p}^{1}, \ldots, \mathbf{p}^{n}\right)
$$

and such that (almost) all agents maximize their payoffs, this meaning that, for each type $i=1, \ldots, n$, all payoffs $r_{j}^{i} \pi_{j}$ are equal to some $C^{i}$ (independent of $j$ ) whenever $p_{j}^{i} \neq 0$, and they do not exceed $C^{i}$ otherwise.

Even a special case of the model involving just one type of agent and characterized by an efficiency vector $(1,1, \ldots, 1)$ exhibits some interest and it can be interpreted as a simple problem of differentiation of social activities in a uniform society. The more realistic case of many types of agents and more complex structure of coefficients of efficiency corresponds to the situation where certain individuals are more capable to fulfill a job than others and some individuals may simply occur more efficient than others. The total output of this "household" production is somehow distributed among the members of the society. We are interested in determining how a socially stable distribution of activities can be implemented by means of a market mechanism.

To prove the existence of a competitive equilibrium for the economy, we construct an auxiliary LSF-game, with $n+1$ types of players, each of whom has $k$ available actions. A generic element of $\left(\Delta_{k}\right)^{n+1}$ is denoted by $\left(\mathbf{p}^{1}, \ldots, \mathbf{p}^{n} ; \pi\right)$. The functions $\Phi^{i}$ composing the game, given by

$$
\begin{aligned}
\Phi^{i}\left(j ; \mathbf{p}^{1}, \ldots, \mathbf{p}^{n} ; \pi\right) & :=r_{j}^{i} \pi_{j} \quad \text { for } i=1, \ldots, n \text { and } j=1, \ldots, k, \\
\Phi^{n+1}\left(j ; \mathbf{p}^{1}, \ldots, \mathbf{p}^{n} ; \pi\right) & :=d_{j}\left(\mathbf{p}^{1}, \ldots, \mathbf{p}^{n} ; \pi\right)-s_{j}\left(\mathbf{p}^{1}, \ldots, \mathbf{p}^{n}\right) \\
& \text { for } j=1, \ldots, k,
\end{aligned}
$$

are all defined on the product of $\{1, \ldots, k\}$ and $n+1$ copies of the simplex $\Delta_{k}$.

Above, $d_{j}$ denotes the $j$ th coordinate of the vector $\mathbf{d}$, and similarly for $s_{j}$. So every agent-producer is interested in maximizing his income and there are 
also infinitely many small "market ghosts" or "invisible hands" (invisible, because they have the size of a point) with one objective: acting infinitesimally, indicate and influence spots of excessive demand.

We have the following theorem:

7.1. THEOREM. Let $\mathcal{E}$ be an economy with $n$ types of agents.

(a) The equilibria for the auxiliary game are the same as the competitive equilibria for $\mathcal{E}$.

(b) If all demand functions $d^{i}$ are continuous then the auxiliary game has an equilibrium; hence there exists a competitive equilibrium for $\mathcal{E}$.

(c) (Walras Law) If all demand functions $d^{i}$ are continuous and satisfy the condition

$$
d^{i}(I, \pi) \cdot \pi=I \quad \text { for all } I \text { and } \pi \text { in the domain of } d^{i},
$$

then, at any competitive equilibrium $\left(\mathbf{p}^{1}, \ldots, \mathbf{p}^{n} ; \pi\right)$, for all $j=1, \ldots, k$,

$$
\mathbf{d}_{j}\left(\mathbf{p}^{1}, \ldots, \mathbf{p}^{n} ; \pi\right)<\mathbf{s}_{j}\left(\mathbf{p}^{1}, \ldots, \mathbf{p}^{n}\right) \quad \text { implies } \pi_{j}=0 .
$$

(d) If all demand functions $d^{i}$ are continuous and satisfy (4) while all coefficients of efficiency $r_{j}^{i}$ are positive, then, at any competitive equilibrium $\left(\mathbf{p}^{1}, \ldots, \mathbf{p}^{n} ; \pi\right)$,

$$
\mathbf{d}_{j}\left(\mathbf{p}^{1}, \ldots, \mathbf{p}^{n} ; \pi\right)=\mathbf{s}_{j}\left(\mathbf{p}^{1}, \ldots, \mathbf{p}^{n}\right) .
$$

If $\pi_{j}=0$ for some $j$, then $\mathbf{s}_{j}\left(\mathbf{p}^{1}, \ldots, \mathbf{p}^{n}\right)=0$.

Proof. (a) By the hypothesis we have, for any equilibrium of the auxiliary game, any $i=1, \ldots, n$ and any $j=1, \ldots, k$,

$$
\left\langle d^{i}\left(r_{j}^{i} \pi_{j}, \pi\right) ; \pi\right\rangle \leq r_{j}^{i} \pi_{j}
$$

and hence

$$
\begin{aligned}
\mathbf{d}_{j}\left(\mathbf{p}^{1}, \ldots, \mathbf{p}^{n} ; \pi\right)=\sum_{i=1}^{n} q^{i} \sum_{j=1}^{k}\left\langle d^{i}\left(r_{j}^{i} \pi_{j}, \pi\right) ; \pi\right\rangle \cdot p_{j}^{i} \\
\quad \leq \sum_{i=1}^{n} q^{i} \sum_{j=1}^{k} r_{j}^{i} \pi_{j} \cdot p_{j}^{i}=\left(\sum_{i=1}^{n} q^{i} \mathbf{r}^{i} \cdot \mathbf{p}^{i}\right) \leq \mathbf{s}_{j}\left(\mathbf{p}^{1}, \ldots, \mathbf{p}^{n}\right) .
\end{aligned}
$$

By the definition of equilibria, for all $j=1, \ldots, k$ with $\pi_{j} \neq 0$, $\mathbf{d}_{j}\left(\mathbf{p}^{1}, \ldots, \mathbf{p}^{n} ; \pi\right)-\mathbf{s}_{j}\left(\mathbf{p}^{1}, \ldots, \mathbf{p}^{n}\right)$ is constant and equal to some $C$. By (5), $C$ cannot be positive, hence $\mathbf{d}_{j}(\mathbf{p} ; \pi) \leq \mathbf{s}_{j}(\mathbf{p})$. If $\pi_{j}=0$, then $\mathbf{d}_{j}(\mathbf{p} ; \pi)-\mathbf{s}_{j}(\mathbf{p})$ does not exceed $C$ either, hence also in this case $\mathbf{d}_{j}(\mathbf{p} ; \pi) \leq \mathbf{s}_{j}(\mathbf{p})$.

(b) An equilibrium for the auxiliary game exists by Theorem 2.1; then we use (a).

(c) Notice that under (4), the inequality (5) becomes an equality.

(d) Notice that $\pi_{j}=0$ implies that (almost) no agent will decide to produce the $j$ th commodity. 
The model describes a simple economy in which only labor is used as input while the capital and primary resources are not involved in a direct manner, although their indirect engagement can be captured by an appropriate description of the types of agents. It can be understood that agents with higher production capacity have an opportunity to invest some capital or use some resources unavailable to agents who are less efficient. A diversification in individual efficiency in various types of activity may also correspond to personal skills, education, etc. In some cases, and this may be typical, certain types $i$ may be unable to undertake some types $j$ of activity, in which case the corresponding coefficient of efficiency $r_{j}^{i}$ is zero.

The model includes some elements already existing in many models arising from the Arrow-Debreu model [1954] (as well as in their original model) and in other works involving a continuum of agents, like those of Aumann [1964], [1966] and Vind [1964] (cf. also a direct application of anonymous games to model competitive markets by Green [1984] or a paper of GarciaCutrin and Hervés-Beloso [1993], including a finitary approach to economies with infinitely many agents, entirely different from ours). However, unlike most models dealing with similar questions, we have not introduced a single entity aimed to reflect the influence of market clearing powers, but rather an infinity of small subjects who eventually elaborate a market clearing price system, but this is done in a decentralized manner as a result of actions performed independently and often with different targets in their range of vision. Efficiency in the presented model was studied by Roman (Ekes) and Wieczorek [1999], core and quasi-core by Ekes [2003], and general aspects by Wieczorek [2002].

While looking at some inequalities in the proof of Theorem 7.1, we find two sorts of units used to measure quantities of each commodity: "small" units which measure individual production or consumption, and "large" ones used to measure the aggregated supply and demand, so the sense of the expressions like " $d_{j}^{i}(I, \pi) \cdot \pi_{j}$ " and " $\mathbf{d}_{j}\left(\mathbf{p}^{1}, \ldots, \mathbf{p}^{n} ; \pi\right) \cdot \pi_{j}$ " is entirely different. The price of an "infinitesimal" unit of quantity intuitively differs from the price of an "integrated" unit but notice that in our considerations it is only the proportion of prices of different goods that matters and these proportions remain unchanged when passing from "infinitesimal" to "integrated" quantities. An extensive discussion of the conceptual meaning of individual behavior in models with a continuum of agents accompanied the introduction of these models in the sixties and there is no reason to repeat more arguments here.

Things do not change much in the model presented in this section if we assume that the potential activities of the agents are not described by their production capacities $r_{j}^{i}$ to produce just one type $j$ of commodity, but they 
are rather described by a finite number, $t^{i}$ for the type $i$, of possible output vectors: $r^{i 1}=\left(r_{1}^{i 1}, \ldots, r_{k}^{i 1}\right), \ldots,\left(r_{1}^{i t^{i}}, \ldots, r_{k}^{i t^{i}}\right)$.

Continuity of an individual demand function $d^{i}$ on the boundary of the price simplex is natural in real life terms and equally hard to capture by means of analytic formulas; the problem is usually neglected by most of the authors, including the pioneers of the subject, Scarf and Hansen [1973], who wrote on p. 20: We shall make this assumption in our discussion of computational procedures even though many of our numerical examples will employ utility functions ... for which the assumption is not valid. The reader should have no difficulty in adjusting to this slight ambiguity. A way to avoid this ambiguity is to use appropriate demand functions instead of utility functions generating them (see Ekes and Wieczorek [2003]).

Acknowledgements. I wish to thank Maria Ekes, Adam Idzik, Tadeusz Radzik and Agnieszka Wiszniewska-Matyszkiel for comments and suggestions concerning the subject matter of the paper.

\section{References}

K. Arrow and G. Debreu [1954], Existence of an equilibrium for a competitive economy, Econometrica 22, 165-290.

R. Aumann [1964], Markets with a continuum of traders, ibid. 32, 39-50.

R. Aumann [1966], Existence of equilibria in markets with a continuum of traders, ibid. 34, $1-17$.

E. Balder [1990], On Cournot-Nash equilibrium distributions for games with differential information and discontinuous payoffs, Economic Theory 1, 339-354.

E. Balder [1995], A unifying approach to existence of Nash equilibria, Int. J. Game Theory 24, 79-94.

R. Cressman [1995], Evolutionary game theory with two groups of individuals, Games Econom. Behav. 11, 237-253.

T. M. Doup, A. H. van der Elzen and A. J. J. Talman [1987], Simplicial algorithm for solving the nonlinear complementarity problem on the simplotope, in: The Computation and Modelling of Economic Equilibria, A. J. J. Talman and G. van der Laan (eds.), North-Holland, 125-153.

T. M. Doup, G. van der Laan and A. J. J. Talman [1987], The $\left(2^{n+1}-2\right)$-ray algorithm: a new simplicial algorithm to compute economic equilibria, Math. Program. 39, 241-252.

T. M. Doup and A. J. J. Talman [1983], A continuous deformation algorithm on the product space of unit simplices, Math. Oper. Res. 12, 485-521.

T. M. Doup and A. J. J. Talman [1987], A new simplicial variable dimension algorithm to find equilibria on the product space of unit simplices, Math. Program. 37, 319-355.

M. Ekes [2003], Core and equilibria in models of Large household economy, Appl. Math. (Warsaw) 30, 431-440.

M. Ekes and A. Wieczorek [2003], Consumption in economic models related to large games, Report \# 956, Institute of Computer Science, Polish Academy of Sciences.

S. D. Flåm and A. Wieczorek [1995], Core solutions and Nash equilibria in non-cooperative games with a measure space of players, Working paper 2495, Department of Economics, University of Bergen. 
J. Garcia-Cutrin and C. Hervés-Beloso [1993], A discrete approach to continuum economies, Econom. Theory 3, 577-583.

E. Green [1984], Continuum and finite-player noncooperative models of competition, Econometrica 52, 975-993.

S. Hart, W. Hildenbrand and E. Kohlberg [1974], On equilibrium allocations as distributions on the commodity space, J. Math. Econom. 1, 159-167.

G. Isac [1992], Complementarity Problems, Lecture Notes in Math. 1528, Springer.

M. A. Khan [1985], On extensions of the Cournot-Nash theorem, in: C. D. Aliprantis, O. Burkinshaw and N. J. Rothman (eds.), Advances in Equilibrium Theory, Lecture Notes in Econom. and Math. Systems 244, Springer, 79-106.

M. A. Khan [1989], On Cournot-Nash equilibrium distributions for games with a nonmetrizable action space and upper semi-continuous payoffs, Trans. Amer. Math. Soc. $315,127-146$.

M. A. Khan and A. Rustichini [1991], Cournot-Nash equilibrium distributions for games with differential information, in: M. A. Théra and J.-B. Baillon (eds.), Fixed Point Theory and Applications (Marseille 1989), Pitman Res. Notes Math. Ser. 252, Longman, 245-259.

M. A. Khan and A. Rustichini [1993], Cournot-Nash equilibrium distributions of games with uncertainty and imperfect information, J. Math. Econom. 22, 35-59.

M. A. Khan and Y. N. Sun [1991], On symmetric Cournot-Nash equilibrium distributions in a finite-action atomless game, in: M. A. Khan and N. C. Yannelis (eds.), Equilibrium Theory in Infinite Dimensional Spaces, Springer, 325-332.

C. E. Lemke and J. T. Howson Jr. [1964], Equilibrium points and bimatrix games, SIAM J. Appl. Math. 12, 413-423.

M. Malawski [1989], Some learning processes in population games, thesis, Department of Economics, Bonn Univ.

A. Mas-Colell [1984], On a theorem of Schmeidler, J. Math. Econom. 13, 201-206.

J. Maynard Smith [1982], Evolution and the Theory of Games, Cambridge Univ. Press.

H. Nikaido and K. Isoda [1955], Note on non-cooperative convex games, Pacific J. Math. 5, 807-815.

K. Rath [1992], A direct proof of the existence of pure strategy equilibria in games with a continuum of players, Econom. Theory 2, 427-433.

K. Rath [1995], Representation of finite action large games, Int. J. Game Theory 24, $23-35$.

K. Ritzberger and J. Weibull [1995], Evolutionary selection in normal form games, Econometrica 63, 1371-1399.

M. Roman (Ekes) and A. Wieczorek [1999], On a multi-objective optimization problem arising from production theory, Appl. Math. (Warsaw) 25 (4), 411-415.

H. Scarf and T. Hansen [1973], The Computation of Economic Equilibria, Yale Univ. Press.

D. Schmeidler [1973], Equilibrium points of nonatomic games, J. Statist. Phys. 17, 295300.

P. Schuster, K. Sigmund, J. Hofbauer, R. Gottlieb and P. Merz [1983], Self-regulation of behaviour in animal societies III, J. Theoret. Biol. 84, 93-101.

P. D. Taylor [1979], Evolutionary stable strategies with two types of players, J. Appl. Probab. 16, 76-83.

G. Van der Laan and A. J. J. Talman [1982], On the computation of fixed points in the product space of unit simplices and an application to noncooperative $N$-person games, Math. Oper. Res. 7, 1-13. 
G. Van der Laan, A. J. J. Talman and L. van der Heyden [1987], Simplicial variable dimension algorithms for solving the nonlinear complementarity problem on a product of unit simplices using a general labelling, Math. Oper. Res. 12, 377-397.

K. Vind [1964], Edgeworth-allocations in an exchange economy with many traders, Int. Econom. Rev. 5, 165-177.

J. Weibull [1995], Evolutionary Game Theory, MIT Press.

A. Wieczorek [1996], Elementary large games and an application to economies with many agents, Report \# 805, Institute of Computer Science, Polish Academy of Sciences.

A. Wieczorek [2002], Household economies with infinitely many agents: a game-theoretic analysis, Int. Game Theory Rev. 4, 319-329.

Institute of Computer Science

Polish Academy of Sciences

Ordona 21

01-237 Warszawa, Poland

E-mail: aw@ipipan.waw.pl

Received on 18.4.2003;

revised version on 24.9 .2003 